\title{
Correction to: Multistage thermal decomposition in films of cadmium chloride-doped PVA-PVP polymeric blend
}

\author{
Basavarajeshwari M. Baraker $^{1}$ Blaise Lobo ${ }^{1}$
}

Published online: 12 May 2018

(C) Akadémiai Kiadó, Budapest, Hungary 2018

\section{Correction to: Journal of Thermal Analysis and Calorimetry https://doi.org/10.1007/s10973-018-7289-5}

There is an error in position of a multiplication factor in Table 2 of the article. The multiplication factor should come directly under the symbol $A$. The corrected table is given as follows (Table 2).

Table 2 Estimated kinetic parameters from the FWO, KAS and Kissinger models at four thermal decomposition stages

\begin{tabular}{|c|c|c|c|c|c|c|c|c|}
\hline \multirow[t]{2}{*}{$\alpha$} & \multicolumn{2}{|l|}{ Second stage } & \multicolumn{2}{|l|}{ Third stage } & \multicolumn{2}{|l|}{ Fourth stage } & \multicolumn{2}{|l|}{ Fifth stage } \\
\hline & $E_{\mathrm{a}} / \mathrm{kJ} \mathrm{mol}^{-1}$ & $A / \min ^{-1}$ & $E_{\mathrm{a}} / \mathrm{kJ} \mathrm{mol}^{-1}$ & $A / \min ^{-1}$ & $E_{\mathrm{a}} / \mathrm{kJ} \mathrm{mol}^{-1}$ & $A / \min ^{-1}$ & $E_{\mathrm{a}} / \mathrm{kJ} \mathrm{mol}^{-1}$ & $A / \min ^{-1}$ \\
\hline \multicolumn{9}{|l|}{ FWO model } \\
\hline & & $\times 10^{4}$ & & $\times 10^{14}$ & & $\times 10^{8}$ & & $\times 10^{7}$ \\
\hline 0.1 & $59.58 \pm 0.17$ & 3.01 & $216.68 \pm 0.11$ & 27.06 & $151.46 \pm 0.07$ & 2.648 & $87.83 \pm 0.05$ & 3.029 \\
\hline 0.3 & $69.31 \pm 0.16$ & 43.88 & $206.81 \pm 0.07$ & 10.03 & $162.29 \pm 0.05$ & 18.492 & $91.57 \pm 0.07$ & 15.96 \\
\hline 0.5 & $79.17 \pm 0.15$ & 162.1 & $196.26 \pm 0.04$ & 1.969 & $187.54 \pm 0.05$ & 535.55 & $93.90 \pm 0.10$ & 34.94 \\
\hline 0.7 & $86.11 \pm 0.14$ & 1284 & $188.19 \pm 0.02$ & 0.524 & $203.09 \pm 0.05$ & 2950.3 & $95.04 \pm 0.15$ & 50.07 \\
\hline 0.9 & $88.15 \pm 0.16$ & 1608 & $179.40 \pm 0.03$ & 0.112 & $210.83 \pm 0.09$ & 4528.1 & $95.07 \pm 0.19$ & 52.58 \\
\hline Average & 76.47 & 620.2 & 197.46 & 7.94 & 183.04 & 1607.0 & 92.68 & 31.32 \\
\hline KAS model & & $\times 10^{4}$ & & $\times 10^{14}$ & & $\times 10^{8}$ & & $\times 10^{7}$ \\
\hline 0.1 & $53.19 \pm 0.19$ & 0.640 & $216.40 \pm 0.12$ & 24.86 & $139.68 \pm 0.09$ & 0.3254 & $83.31 \pm 0.05$ & 0.700 \\
\hline 0.3 & $63.15 \pm 0.18$ & 5.261 & $205.92 \pm 0.07$ & 8.234 & $150.70 \pm 0.07$ & 1.7032 & $87.11 \pm 0.08$ & 3.905 \\
\hline 0.5 & $73.28 \pm 0.17$ & 52.99 & $194.73 \pm 0.04$ & 1.429 & $176.29 \pm 0.04$ & 53.622 & $89.43 \pm 0.11$ & 8.770 \\
\hline 0.7 & $80.34 \pm 0.16$ & 220.7 & $186.16 \pm 0.02$ & 0.344 & $192.15 \pm 0.06$ & 557.26 & $90.52 \pm 0.15$ & 12.56 \\
\hline 0.9 & $82.24 \pm 0.18$ & 290.4 & $176.81 \pm 0.03$ & 0.065 & $200.55 \pm 0.11$ & 985.13 & $90.44 \pm 0.19$ & 12.87 \\
\hline Average & 70.44 & 114.0 & 196.00 & 6.986 & 171.88 & 319.61 & 88.16 & 7.76 \\
\hline \multicolumn{9}{|c|}{ Kissinger model } \\
\hline & & $\times 10^{14}$ & & $\times 10^{10}$ & & $\times 10^{4}$ & & $\times 10^{7}$ \\
\hline & $165.12 \pm 0.04$ & 3.22 & $175.26 \pm 0.019$ & 2.000 & $78.27 \pm 0.303$ & 1.19 & $78.52 \pm 0.088$ & 3.36 \\
\hline
\end{tabular}

The symbols $E_{\mathrm{a}}$ and $A$ are used for activation energy and pre-exponential factor, respectively, whereas $E_{\alpha}$ and $A_{\alpha}$ are used (for denoting activation energy and pre-exponential factor) in FWO, KAS and Kissinger equations, respectively

The original article can be found online at https:// doi.org/10.1007/s10973-018-7289-5.

Blaise Lobo

blaise.lobo@gmail.com

1 Department of Physics, Karnatak University's Karnatak Science College, Dharwad, Karnataka 580001, India 